PROCEEDINGS OF THE

AMERICAN MATHEMATICAL SOCIETY

Volume 135, Number 10, October 2007, Pages 3099-3108

S 0002-9939(07)08873-9

Article electronically published on June 20, 2007

\title{
$L$-FUNCTIONS \\ OF TWISTED DIAGONAL EXPONENTIAL SUMS OVER FINITE FIELDS
}

\author{
SHAOFANG HONG \\ (Communicated by Wen-Ching Winnie Li)
}

Abstract. Let $\mathbf{F}_{q}$ be the finite field of $q$ elements with characteristic $p$ and $\mathbf{F}_{q^{m}}$ its extension of degree $m$. Fix a nontrivial additive character $\Psi$ and let $\chi_{1}, \ldots, \chi_{n}$ be multiplicative characters of $\mathbf{F}_{p}$. For

$$
f\left(x_{1}, \ldots, x_{n}\right) \in \mathbf{F}_{q}\left[x_{1}, x_{1}^{-1}, \ldots, x_{n}, x_{n}^{-1}\right],
$$

one can form the twisted exponential sum $S_{m}^{*}\left(\chi_{1}, \ldots, \chi_{n}, f\right)$. The corresponding $L$-function is defined by

$$
L^{*}\left(\chi_{1}, \ldots, \chi_{n}, f ; t\right)=\exp \left(\sum_{m=0}^{\infty} S_{m}^{*}\left(\chi_{1}, \ldots, \chi_{n}, f\right) \frac{t^{m}}{m}\right)
$$

In this paper, by using the $p$-adic gamma function and the Gross-Koblitz formula on Gauss sums, we give an explicit formula for the $L$-function $L^{*}\left(\chi_{1}, \ldots, \chi_{n}, f ; t\right)$ if $f$ is a Laurent diagonal polynomial. We also determine its $p$-adic Newton polygon.

\section{INTRODUCTION}

Let $\mathbf{F}_{q}$ be the finite field of $q$ elements with characteristic $p$ and $\mathbf{F}_{q^{m}}$ its extension of degree $m$. Fix a nontrivial additive character $\Psi$ and let $\chi_{1}, \ldots, \chi_{n}$ be multiplicative characters of $\mathbf{F}_{q}$. If $f\left(x_{1}, \ldots, x_{n}\right) \in \mathbf{F}_{q}\left[x_{1}, x_{1}^{-1}, \ldots, x_{n}, x_{n}^{-1}\right]$, then one can form the twisted exponential sum

$$
\begin{aligned}
S_{m}^{*}\left(\chi_{1}, \ldots, \chi_{n}, f\right) & \sum_{\left(x_{1}, \ldots, x_{n}\right) \in\left(\mathbf{F}_{q^{m}}^{*}\right)^{n}} \chi_{1}\left(\mathrm{~N}_{m}\left(x_{1}\right)\right) \ldots \chi_{n}\left(\mathrm{~N}_{m}\left(x_{n}\right)\right) \Psi\left(\operatorname{Tr}_{m}\left(f\left(x_{1}, \ldots, x_{n}\right)\right)\right),
\end{aligned}
$$

where $\operatorname{Tr}_{m}: \mathbf{F}_{q^{m}} \rightarrow \mathbf{F}_{q}$ is the trace map and $\mathrm{N}_{m}: \mathbf{F}_{q^{m}} \rightarrow \mathbf{F}_{q}$ is the norm map. The corresponding $L$-function is defined as follows:

$$
L^{*}\left(\chi_{1}, \ldots, \chi_{n}, f ; t\right)=\exp \left(\sum_{m=0}^{\infty} S_{m}^{*}\left(\chi_{1}, \ldots, \chi_{n}, f\right) \frac{t^{m}}{m}\right) \text {. }
$$

Received by the editors May 1, 2006 and, in revised form, July 20, 2006.

2000 Mathematics Subject Classification. Primary 11L03, 11T23, 14 G10.

Key words and phrases. Twisted exponential sum, L-function, $p$-adic Newton polygon, $\Gamma$ function, Gross-Koblitz formula, Hasse-Davenport relation.

The research of this author was supported by New Century Excellent Talents in University Grant \# NCET-060785, by SRF for ROCS, SEM and by NNSF of China Grant \# 10101015. 
By the renowned Dwork-Grothendieck theorem, one knows that the $L$-function $L^{*}\left(\chi_{1}, \ldots, \chi_{n}, f ; t\right)$ is a rational function.

Finding sharp $p$-adic estimates of the exponential sums $S_{m}^{*}\left(\chi_{1}, \ldots, \chi_{n}, f\right)$ is a fundamental question in number theory. This is well known to be reduced to determining the $p$-adic absolute values of the reciprocal roots and reciprocal poles. The best way to describe this problem is in terms of $p$-adic Newton polygons (see [11). Of course one may consider the question of finding the $p$-adic Newton polygon of Dwork's unit root $L$-function attached to a family of algebraic varieties over $\mathbf{F}_{q}$, which is proved by Wan $18,19,20$, to be $p$-adic meromorphic as conjectured by Dwork [4. Such question should be very deep and hard.

On the other hand, Dwork [5] determined the Newton polygon for the $L$-functions of the one-dimensional Kloosterman sum, and then Sperber [13] generalized Dwork's result to the $n$-dimensional case while Sperber [14] considered the general hyperKloosterman sums. Wan [16, 17, 21] solved a conjecture of Adolphson and Sperber [1. Note also that Wan [17] presented a $p$-adic proof of the Dwork-Mazur conjecture [3, 11. When all the $\chi_{i}$ are trivial, then the $p$-adic Newton polygon of the $L$-function of the one-variable polynomial $f(x)$ received attention by Sperber [15, Hong [7, 8, 9] and Yang 22. A general interesting question is to determine the $p$-adic Newton polygon of the $L$-function $L^{*}(\chi, f ; t)$ if $f$ is of one variable, even for small degree, and $\chi$ is a nontrivial multiplicative character.

In this paper, we are interested in the case that $f\left(x_{1}, \ldots, x_{n}\right)$ is a Laurent diagonal polynomial. We obtain an explicit formula for the $L$-function $L^{*}\left(\chi_{1}, \ldots, \chi_{n}, f ; t\right)$ if $f$ is a Laurent diagonal polynomial by using the $p$-adic gamma function. We also determine the $p$-adic Newton polygon for such an $L$-function $L^{*}\left(\chi_{1}, \ldots, \chi_{n}, f ; t\right)$. In the forthcoming works, we will use the results presented here to investigate a conjecture of Adolphson and Sperber [2] on the p-adic Newton polygons of twisted exponential sums.

\section{Preliminaries on $p$-ADiC Gamma funCtions}

In this section, we present some properties of the $p$-adic gamma function $\Gamma_{p}$. For $s \in \mathbf{Z}^{+}$one sets

$$
\Gamma(s)=(-1)^{s} \cdot \prod_{i=1}^{s-1} i .
$$

It is known (see, for example, 12]) that this extends to a continuous function on $\mathbf{Z}_{p}$, and we define $\Gamma_{p}$ to be this extension. Therefore it follows from continuity that the following functional equation holds:

$$
\frac{\Gamma_{p}(x+1)}{\Gamma_{p}(x)}= \begin{cases}-1, & \text { if } x \in p \mathbf{Z}_{p}, \\ -x, & \text { if } x \notin p \mathbf{Z}_{p}\end{cases}
$$

and also we have

$$
\Gamma_{p}(x) \Gamma_{p}(1-x)=(-1)^{\langle x\rangle_{p}},
$$

where $\langle x\rangle_{p}$ denotes the least nonnegative residue of $x$ modulo $p$.

Denote by $\zeta_{p}$ a fixed primitive $p$-th root of unity in $\mathbf{C}_{p}$, the completion of the algebraic closure of the $p$-adic field $\mathbf{Q}_{p}$. Then there is a generator $\pi$ of the maximal ideal of the ring of integers of $\mathbf{Q}_{p}\left(\zeta_{p}\right)$ uniquely characterized by $\pi^{p-1}=-p$ and $\pi \equiv \zeta_{p}-1\left(\bmod \left(\zeta_{p}-1\right)^{2}\right)$. Let $\varpi$ be any fixed Teichmüller character of $\mathbf{F}_{q}$. Let $\sigma_{p}(a)$ denote the sum of the digits of the $p$-adic representation of an integer $a \geq 1$. 
Lemma 2.1 (Gross-Koblitz formula, see [6] or [12]). Let $1 \leq a<p^{e}:=q$. Then the value of the Gauss sum $g_{q}(a)$ is explicitly given by

$$
g_{q}(a):=-\sum_{0 \neq x \in \mathbf{F}_{q}} \omega(x)^{-a} \Psi(\operatorname{Tr}(x))=\pi^{\sigma_{p}(a)} \prod_{j=0}^{e-1} \Gamma_{p}\left(\frac{\left\langle p^{j} a\right\rangle_{q-1}}{q-1}\right) .
$$

\section{TWisted Diagonal EXPONEntial sums}

For brevity, we define $x^{\alpha}:=x_{1}^{\alpha_{1}} \ldots x_{n}^{\alpha_{n}}$ for any vector $x=\left(x_{1}, \ldots, x_{n}\right) \in\left(\mathbf{F}_{q^{m}}^{*}\right)^{n}$ and any $n$-tuple $\alpha=\left(\alpha_{1}, \ldots, \alpha_{n}\right) \in \mathbf{Z}^{n}$. Since $\zeta_{p}$ is a given primitive $p$ th root of unity in $\mathbf{C}_{p}$, we know that for any given nontrivial additive character $\Psi$ of $\mathbf{F}_{q}$, we have $\Psi(c)=\zeta_{p}{ }^{\operatorname{Tr}_{\mathbf{F}_{q} / \mathbf{F}_{p}}(c)}, c \in \mathrm{F}_{q}$. Let $\varpi_{l}$ be any fixed Teichmüller character of $\mathbf{F}_{q^{l}}$ for any given integer $l \geq 1$. Then $\varpi_{1}=\varpi$ and for multiplicative characters $\chi_{1}, \ldots, \chi_{n}$, there must be nonnegative integers $b_{1}, \ldots, b_{n}$, such that $\chi_{i}=\varpi^{b_{i}}, 1 \leq i \leq n$. Thus we can rewrite (1.1) as follows:

$$
S_{m}^{*}\left(\chi_{1}, \ldots, \chi_{n}, f\right)=\sum_{x \in\left(\mathbf{F}_{q}^{*}\right)^{n}} \zeta_{p}^{\operatorname{Tr}_{\mathbf{F}_{q} m / \mathbf{F}_{p}}(f(x))} \cdot \prod_{i=1}^{n}\left(\varpi^{b_{i}} \circ \mathbf{N}_{m}\right)\left(x_{i}\right) .
$$

Lemma 3.1. For any $c \in \mathbf{F}_{q}$ and any integer $l \geq 1$, we have $\varpi_{l}(c)=\varpi(c)$.

Proof. For any positive integer $c$, let $\mathbf{Z}_{c}^{\text {unram }}$ denote the ring of integers of the unramified extension of $\mathbf{Q}_{p}$ of degree $c$. Since the diagram

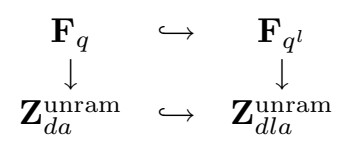

commutes, the result follows immediately. The proof is complete.

Now for $x_{i} \in \mathbf{F}_{q^{m}}^{*}$, we have

$$
\left(\varpi^{b_{i}} \circ \mathrm{N}_{m}\right)\left(x_{i}\right)=\varpi^{b_{i}}\left(x_{i}^{\frac{q^{m}-1}{q-1}}\right)=\varpi\left(x_{i}^{\frac{q^{m}-1}{q-1} \cdot b_{i}}\right) .
$$

Since $x_{i} \in \mathbf{F}_{q^{m}}^{*}$, we have $x_{i}^{\frac{q^{m}-1}{q-1} \cdot b_{i}} \in \mathbf{F}_{q}$. By Lemma 3.1 we obtain

$$
\left(\varpi^{b_{i}} \circ \mathrm{N}_{m}\right)\left(x_{i}\right)=\varpi_{m}\left(x_{i}^{\frac{q^{m}-1}{q-1} \cdot b_{i}}\right)
$$

So by (3.1) our sum (1.1) may be rewritten as

$$
S_{m}^{*}\left(\chi_{1}, \ldots, \chi_{n}, f\right)=\sum_{x \in\left(\mathbf{F}_{q}^{*}\right)^{n}} \zeta_{p}^{\operatorname{Tr}_{\mathbf{F}_{q} m} / \mathbf{F}_{p}(f(x))} \cdot \varpi_{m}\left(x^{\frac{q^{m}-1}{q-1} \cdot b}\right),
$$

where $b=\left(b_{1}, \ldots, b_{n}\right)$. From the definition of Gauss sums over $\mathbf{F}_{q^{m}}$, one can deduce that for each $c \in \mathbf{F}_{q^{m}}$, the Gauss sum satisfies the following interpolation relation:

$$
\zeta_{p}^{\operatorname{Tr}_{\mathbf{F}_{q} / \mathbf{F}_{p}}(c)}=\frac{1}{1-q^{m}} \sum_{k=0}^{q^{m}-2} g_{q^{m}}(k) \cdot \varpi_{m}(c)^{k} .
$$


Now let $f(x)=f\left(x_{1}, \ldots, x_{n}\right)=\sum_{i=1}^{n} a_{i} x^{V_{i}} \in \mathbf{F}_{q}\left[x_{1}, x_{1}^{-1}, \ldots, x_{n}, x_{n}^{-1}\right]$ be a diagonal polynomial. Then by (3.2) and (3.3) we derive that

$$
\begin{aligned}
& S_{m}^{*}\left(\chi_{1}, \ldots, \chi_{n}, f\right)=\sum_{x \in\left(\mathbf{F}_{q}^{*}\right)^{n}} \zeta_{p}^{\operatorname{Tr}_{\mathbf{F}_{q} / \mathbf{F}_{p}}\left(\sum_{i=1}^{n} a_{i} x^{V_{i}}\right)} \cdot \varpi_{m}\left(x^{\frac{q^{m}-1}{q-1} \cdot b}\right) \\
& =\sum_{x \in\left(\mathbf{F}_{q^{m}}^{*}\right)^{n}} \varpi_{m}\left(x^{\frac{q^{m}-1}{q-1} \cdot b}\right) \cdot \prod_{i=1}^{n} \zeta_{p}^{\operatorname{Tr}_{\mathbf{F}_{q} m / \mathbf{F}_{p}}\left(a_{i} x^{V_{i}}\right)} \\
& =\sum_{x \in\left(\mathbf{F}_{q^{m}}^{*}\right)^{n}} \varpi_{m}\left(x^{\frac{q^{m}-1}{q-1} \cdot b}\right) \cdot \prod_{i=1}^{n} \frac{1}{1-q^{m}} \sum_{k_{i}=0}^{q^{m}-2} g_{q^{m}}\left(k_{i}\right) \cdot \varpi_{m}\left(a_{i} x^{V_{i}}\right)^{k_{i}} \\
& =\sum_{x \in\left(\mathbf{F}_{q^{m}}^{*}\right)^{n}} \varpi_{m}\left(x^{\frac{q^{m}-1}{q-1} \cdot b}\right) \cdot \sum_{k_{1}=0}^{q^{m}-2} \ldots \sum_{k_{n}=0}^{q^{m}-2} \prod_{i=1}^{n} \frac{g_{q^{m}}\left(k_{i}\right)}{1-q^{m}} \cdot \varpi_{m}\left(a_{i} x^{V_{i}}\right)^{k_{i}} \\
& =\sum_{k_{1}=0}^{q^{m}-2} \ldots \sum_{k_{n}=0}^{q^{m}-2} \sum_{x \in\left(\mathbf{F}_{q^{m}}^{*}\right)^{n}} \varpi_{m}\left(x^{\frac{q^{m}-1}{q-1} \cdot b}\right) \prod_{i=1}^{n} \frac{g_{q^{m}}\left(k_{i}\right)}{1-q^{m}} \cdot \varpi_{m}\left(a_{i} x^{V_{i}}\right)^{k_{i}} \\
& =\sum_{k_{1}=0}^{q^{m}-2} \ldots \sum_{k_{n}=0}^{q^{m}-2}\left(\prod_{i=1}^{n} \frac{g_{q^{m}}\left(k_{i}\right)}{1-q^{m}} \cdot \varpi_{m}\left(a_{i}\right)^{k_{i}}\right) \cdot \sum_{x \in\left(\mathbf{F}_{q^{m}}^{*}\right)^{n}} \varpi_{m}\left(x^{\sum_{i=1}^{n} k_{i} V_{i}+\frac{q^{m}-1}{q-1} \cdot b}\right) .
\end{aligned}
$$

Noting that

$$
\sum_{x \in\left(\mathbf{F}_{q}^{*}\right)^{n}} \varpi_{m}\left(x^{\sum_{i=1}^{n} k_{i} V_{i}+\frac{q^{m}-1}{q-1} \cdot b}\right)
$$

is equal to $\left(q^{m}-1\right)^{n}$ if $\sum_{i=1}^{n} k_{i} V_{i}+\frac{q^{m}-1}{q-1} \cdot b \equiv 0\left(\bmod q^{m}-1\right)$, and 0 otherwise, one then deduces that

$$
\begin{aligned}
& S_{m}^{*}\left(\chi_{1}, \ldots, \chi_{n}, f\right)=\sum_{\substack{\sum_{i=1}^{n} k_{i} V_{i}+\frac{q^{m}-1}{q-1} \cdot b \equiv 0 \\
0 \leq k_{i} \leq q^{m}-2 \text { for }\left(\bmod q^{m}-1\right)}}\left(q^{m}-1\right)^{n} \prod_{i=1}^{n} \frac{g_{q^{m}}\left(k_{i}\right)}{1-q^{m}} \cdot \varpi_{m}\left(a_{i}\right)^{k_{i}} \\
&=(-1)^{n} \cdot \sum_{\substack{\sum_{i=1}^{n} k_{i} V_{i}+\frac{q^{m}-1}{q-1} \cdot b \equiv 0\left(\bmod q^{m}-1\right) \\
0 \leq k_{i} \leq q^{m}-2 \text { for } 1 \leq i \leq n}}^{n} \prod_{\substack{i=1 \\
(3.4)}}^{n} g_{q^{m}}\left(k_{i}\right) \cdot \varpi_{m}\left(a_{i}\right)^{k_{i}} .
\end{aligned}
$$

Let each $V_{i}$ be written as a column vector, and let $M=\left(V_{1}, \ldots, V_{n}\right)$ be the $n \times n$ matrix having $V_{i}$ as its $i$-th column for $1 \leq i \leq n$. Write $u_{i}=\frac{k_{i}}{q^{m}-1}$ and $b_{i}^{\prime}=\frac{b_{i}}{q-1}$ for $1 \leq i \leq n$. Then for all $1 \leq i \leq n$, we have $0 \leq u_{i}, b_{i}^{\prime}<1$. It is clear that

$$
\sum_{i=1}^{n} k_{i} V_{i}+\frac{q^{m}-1}{q-1} \cdot b \equiv 0\left(\bmod q^{m}-1\right)
$$

if and only if

$$
M u+b^{\prime} \equiv 0(\bmod 1),
$$

where $u=\left(k_{1}^{\prime}, \ldots, k_{n}^{\prime}\right)^{T}$ and $b^{\prime}=\left(b_{1}^{\prime}, \ldots, b_{n}^{\prime}\right)^{T}$ are written as column vectors. Then from (3.4) we finish the proof of the following result.

Theorem 3.2. For any integer $m \geq 1$, we have

$$
S_{m}^{*}\left(\chi_{1}, \ldots, \chi_{n}, f\right)=(-1)^{n} \cdot \sum_{\substack{M u+b^{\prime} \equiv 0(\bmod 1) \\\left(q^{m}-1\right) u \in \mathbf{Z}^{n}}} \prod_{i=1}^{n} g_{q^{m}}\left(u_{i}\left(q^{m}-1\right)\right) \cdot \varpi_{m}\left(a_{i}\right)^{u_{i}\left(q^{m}-1\right)} .
$$




\section{Explicit FORMulas of $L$-FUnCtions of TWISTED DiAgOnAL SUMS AND NEWTON POLYGONS}

In this section, we will give an explicit formula for the $L$-function of the twisted sum of the diagonal Laurent polynomial over $\mathbf{F}_{q}$. Let $f(x)=\sum_{i=1}^{n} a_{i} x^{V_{i}}$ be a diagonal Laurent polynomial and $\Delta$ the Newton polyhedron generated by the $n$ indices $V_{i}, 1 \leq i \leq n$. Let $S\left(\Delta, b^{\prime}\right)$ be the set of solutions $u$ of the following linear system of congruences:

$$
M u+b^{\prime} \equiv 0(\bmod 1), u_{i} \text { rational numbers, } 0 \leq u_{i}<1 .
$$

Now suppose that $(\operatorname{det} M, p)=1$. Then for each $u \in S\left(\Delta, b^{\prime}\right)$, there exists a smallest positive integer, denoted by $d\left(q, b^{\prime}, u\right)$, such that the multiplication by $q^{d\left(q, b^{\prime}, u\right)}$ acts trivially on $u+b^{\prime}$. We claim that this is equivalent to $\left(q^{d\left(q, b^{\prime}, u\right)}-1\right) u \in$ $\mathbf{Z}^{n}$. In fact, since $(q-1) b^{\prime}=b \in \mathbf{Z}^{n}$, we have $q^{d\left(q, b^{\prime}, u\right)}\left(u+b^{\prime}\right) \equiv u+b^{\prime}(\bmod 1) \Leftrightarrow$ $\left(q^{d\left(q, b^{\prime}, u\right)}-1\right) u \equiv\left(1-q^{d\left(q, b^{\prime}, u\right)}\right) b^{\prime}(\bmod 1) \Leftrightarrow\left(q^{d\left(q, b^{\prime}, u\right)}-1\right) u \equiv 0(\bmod 1)$. So the assertion is proved. Therefore $d\left(q, b^{\prime}, u\right)$ is the smallest positive integer $d^{\prime}$ such that $\left(q^{d^{\prime}}-1\right) u \in \mathbf{Z}^{n}$.

For any given integer $d \in \mathbf{Z}^{+}$, let $S\left(q, b^{\prime}, d\right)=\left\{u \in S\left(\Delta, b^{\prime}\right) \mid d\left(q, b^{\prime}, u\right)=d\right\}$. Define a set $I$ of indices by $I:=\left\{d \in \mathbf{Z}^{+} \mid \exists u \in S\left(\Delta, b^{\prime}\right)\right.$, such that $\left.d\left(q, b^{\prime}, u\right)=d\right\}$. Since $S\left(\Delta, b^{\prime}\right)$ is finite, so is $I$.

To give the main result, one first needs two lemmas.

Lemma 4.1. For any integer $l$ and $0 \leq k \leq q^{d}-2$, we have

$$
g_{q^{d l}}\left(k \cdot \frac{q^{d l}-1}{q^{d}-1}\right)=g_{q^{d}}(k)^{l} .
$$

Proof. First we have

$$
g_{q^{d l}}\left(k \cdot \frac{q^{d l}-1}{q^{d}-1}\right)=-\sum_{c \in \mathbf{F}_{q^{*}}^{*}} \varpi_{d l}\left(\mathrm{~N}_{d l}(c)\right)^{-k} \cdot \zeta_{p}^{\operatorname{Tr}_{\mathbf{F}_{q} d l} / \mathbf{F}_{p}(c)} .
$$

Since $\mathrm{N}_{d l}(c) \in \mathbf{F}_{q}$, by Lemma 3.1 we have $\varpi_{d l}\left(\mathrm{~N}_{d l}(c)\right)=\varpi_{d}\left(\mathrm{~N}_{d l}(c)\right)$. Thus

$$
g_{q^{d l}}\left(k \cdot \frac{q^{d l}-1}{q^{d}-1}\right)=-\sum_{c \in \mathbf{F}_{q}^{*} d l} \varpi_{d}^{-k}\left(\mathrm{~N}_{d l}(c)\right) \cdot \zeta_{p}^{\operatorname{Tr}_{\mathbf{F}_{q} d l} / \mathbf{F}_{p}(c)} .
$$

Then it follows from the well-known Hasse-Davenport relation (see, for example, [10]) that

$$
g_{q^{d l}}\left(k \cdot \frac{q^{d l}-1}{q^{d}-1}\right)=\left(-\sum_{c \in \mathbf{F}_{q^{d}}^{*}} \varpi_{d}^{-k}\left(N_{d}(c)\right) \cdot \zeta_{p}^{\operatorname{Tr}_{\mathbf{F}_{q^{d}} / \mathbf{F}_{p}}(c)}\right)^{l}=\left(g_{q^{d}}(k)\right)^{l}=g_{q^{d}}(k)^{l},
$$

as desired. The proof is complete.

Lemma 4.2. The Gauss sum is invariant under $p$-action; i.e., we have $g_{q}(p k)=$ $g_{q}(k)$.

Proof. We have

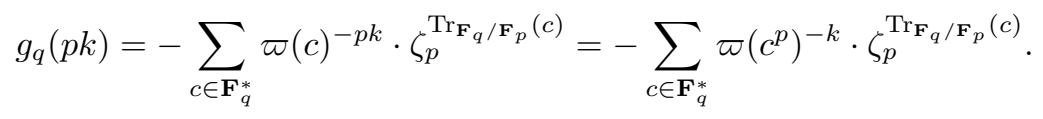


Noting that $\operatorname{Tr}_{\mathbf{F}_{q} / \mathbf{F}_{p}}(c)=\operatorname{Tr}_{\mathbf{F}_{q} / \mathbf{F}_{p}}\left(c^{p}\right)$, we then have

$$
g_{q}(p k)=-\sum_{c \in \mathbf{F}_{q}^{*}} \varpi\left(c^{p}\right)^{-k} \cdot \zeta_{p}^{\operatorname{Tr}_{\mathbf{F}} / \mathbf{F}_{p}\left(c^{p}\right)} .
$$

Let $c^{\prime}=c^{p}$. It is easy to see that if $c$ runs over $\mathbf{F}_{q}^{*}$, then $c^{\prime}$ runs also over $\mathbf{F}_{q}^{*}$. Therefore

$$
g_{q}(p k)=-\sum_{c \in \mathbf{F}_{q}^{*}} \varpi\left(c^{\prime}\right)^{-k} \cdot \zeta_{p}^{\operatorname{Tr}_{\mathbf{F}} / \mathbf{F}_{p}\left(c^{\prime}\right)}=g_{q}(k) .
$$

This completes the proof of Lemma 4.2.

As usual, let $\{r\}$ denote the fractional part of the real number $r$, namely $\{r\}=$ $r-[r]$. For any $u \in S\left(q, b^{\prime}, d\right)$, one can easily check that $\{u\} \in S\left(q, b^{\prime}, d\right)$. So we can define the $q$-action on $u$ by $q \cdot u:=\{q u\}$, where $\{q u\}=\left(\left\{q u_{1}\right\}, \ldots,\left\{q u_{n}\right\}\right)$. Evidently for any $0 \leq i \neq j \leq d-1$, we have $\left\{q^{i} u\right\} \neq\left\{q^{j} u\right\}$ and $\left\{q^{d} u\right\}=u$. Namely $\left\{\left\{q^{l} u\right\} \mid l \in \mathbf{Z}_{\geq 0}\right\}=\left\{u,\{q u\}, \ldots,\left\{q^{d-1} u\right\}\right\}$. Call it an orbit (closed point) of $u$ under $q$-action. Hence there are $d$ distinct points in the orbit of $u \in S\left(q, b^{\prime}, d\right)$ under $q$-action. Then $S\left(q, b^{\prime}, d\right)$ can be partitioned into distinct orbits. We take an element from each orbit of $S\left(q, b^{\prime}, d\right)$ as the representation of this orbit and put these representations together to form a set $O\left(S\left(q, b^{\prime}, d\right)\right)$.

We are now ready to give the main result in this paper.

Theorem 4.3. Let $f(x)=\sum_{i=1}^{n} a_{i} x^{V_{i}} \in \mathbf{F}_{q}\left[x_{1}, x_{1}^{-1}, \ldots, x_{n}, x_{n}^{-1}\right]$ be a diagonal Laurent polynomial, and let $M=\left(V_{1}, \ldots, V_{n}\right)$ be the $n \times n$ matrix having $V_{i}$ as its $i$-th column for $1 \leq i \leq n$. Let $(p, \operatorname{det} M)=1$ and $q=p^{e}$. Then we have

$L^{*}\left(\chi_{1}, \ldots, \chi_{n}, f ; t\right)$

$$
=\prod_{d \in I} \prod_{u \in O\left(S\left(q, b^{\prime}, d\right)\right)}\left(1-t^{d} \cdot \pi^{\sum_{i=1}^{n} \sigma_{p}\left(u_{i}\left(q^{d}-1\right)\right)}\right.
$$

$$
\left.\cdot \prod_{i=1}^{n} \varpi\left(a_{i}\right)^{u_{i}\left(q^{d}-1\right)} \prod_{j=0}^{d e-1} \Gamma_{p}\left(\frac{\left\langle p^{j} u_{i}\left(q^{d}-1\right)\right\rangle_{q^{d}-1}}{q^{d}-1}\right)\right) .
$$

Furthermore, if $u \in O\left(S\left(q, b^{\prime}, d\right)\right)$ and $\omega_{u}$ is any one of the $d$ reciprocal roots of the corresponding factor in (4.1), then we have

$$
\operatorname{ord}_{q} \omega_{u}=\frac{1}{d} \sum_{j=0}^{d e-1} \sum_{i=1}^{n}\left\{p^{j} u_{i}\right\}
$$

Proof. From Theorem 3.2 we deduce that

$L^{*}\left(\chi_{1}, \ldots, \chi_{n}, f ; t\right)$

$$
=\exp \left(\sum_{m=1}^{\infty}(-1)^{n} \cdot \sum_{\substack{u \in S\left(\Delta, b^{\prime}\right) \\\left(q^{m}-1\right) u \in \mathbf{Z}^{n}}} \prod_{i=1}^{n} \varpi_{m}\left(a_{i}\right)^{u_{i}\left(q^{m}-1\right)} \cdot g_{q^{m}}\left(u_{i}\left(q^{m}-1\right)\right) \cdot \frac{t^{m}}{m}\right) .
$$

Clearly, for any given $u$ satisfying $M u+b^{\prime} \equiv 0(\bmod 1)$, there exists a unique integer $d$ such that $u \in S\left(q, b^{\prime}, d\right)$, i.e., such that $\left(q^{d}-1\right) u+b^{\prime} \in \mathbf{Z}^{n}$, but $\left(q^{d-1}-1\right) u+b^{\prime} \notin \mathbf{Z}^{n}$. 
For such a $u$, we have $\left(q^{d k}-1\right) u+b^{\prime} \in \mathbf{Z}^{n}$ for any integer $k \geq 1$. So by (4.2) we have

$L^{*}\left(\chi_{1}, \ldots, \chi_{n}, f ; t\right)$

$$
=\exp \left((-1)^{n} \cdot \sum_{d=1}^{\infty} \sum_{u \in S\left(q, d, b^{\prime}\right)} \prod_{k=1}^{\infty} \prod_{i=1}^{n} \varpi\left(a_{i}\right)^{u_{i}\left(q^{d k}-1\right)} \cdot g_{q^{d k}}\left(u_{i}\left(q^{d k}-1\right)\right) \cdot \frac{t^{d k}}{d k}\right) .
$$

By Lemma 4.1, one knows that for any $u \in S\left(q, b^{\prime}, d\right)$,

$$
g_{q^{d k}}\left(u_{i}\left(q^{d k}-1\right)\right)=g_{q^{d}}\left(u_{i}\left(q^{d}-1\right)\right)^{k} .
$$

Note also that for any $u \in S\left(q, b^{\prime}, d\right)$, we have $\varpi\left(a_{i}\right)^{u_{i}\left(q^{d k}-1\right)}=\varpi\left(a_{i}\right)^{u_{i}\left(q^{d}-1\right) k}$. Then by (4.3) and (4.4) we have

$$
\begin{aligned}
& L^{*}\left(\chi_{1}, \ldots, \chi_{n}, f ; t\right) \\
& =\exp \left((-1)^{n} \cdot \sum_{d=1}^{\infty} \sum_{u \in S\left(q, b^{\prime}, d\right)} \sum_{k=1}^{\infty} \frac{t^{d k}}{d k} \cdot \prod_{i=1}^{n} \varpi\left(a_{i}\right)^{u_{i}\left(q^{d}-1\right) k} \cdot g_{q^{d}}\left(u_{i}\left(q^{d}-1\right)\right)^{k}\right) \\
& =\exp \left(\sum_{d=1}^{\infty} \frac{(-1)^{n-1}}{d} \sum_{u \in S\left(q, b^{\prime}, d\right)} \sum_{k=1}^{\infty}-\frac{\prod_{i=1}^{n} \varpi\left(a_{i}\right)^{u_{i}\left(q^{d}-1\right) k} \cdot g_{q^{d}}\left(u_{i}\left(q^{d}-1\right)\right)^{k} \cdot t^{d k}}{k}\right) \\
& =\exp \left(\sum_{d=1}^{\infty} \frac{(-1)^{n-1}}{d} \sum_{u \in S\left(q, b^{\prime}, d\right)} \log \left(1-t^{d} \prod_{i=1}^{n} \varpi\left(a_{i}\right)^{u_{i}\left(q^{d}-1\right)} \cdot g_{q^{d}}\left(u_{i}\left(q^{d}-1\right)\right)\right)\right) \\
& =\prod_{d=1}^{\infty} \prod_{u \in S\left(q, b^{\prime}, d\right)} \exp \left(\frac{(-1)^{n-1}}{d} \log \left(1-t^{d} \prod_{i=1}^{n} \varpi\left(a_{i}\right)^{u_{i}\left(q^{d}-1\right)} \cdot g_{q^{d}}\left(u_{i}\left(q^{d}-1\right)\right)\right)\right) \\
& =\prod_{d=1}^{\infty} \prod_{u \in S\left(q, b^{\prime}, d\right)}\left(1-t^{d} \prod_{i=1}^{n} \varpi\left(a_{i}\right)^{u_{i}\left(q^{d}-1\right)} \cdot g_{q^{d}}\left(u_{i}\left(q^{d}-1\right)\right)\right)^{\frac{(-1)^{n-1}}{d}}
\end{aligned}
$$

It follows immediately from (4.5) that

$$
\begin{aligned}
& L^{*}\left(\chi_{1}, \ldots, \chi_{n}, f ; t\right)^{(-1)^{n-1}} \\
& =\prod_{d=1}^{\infty} \prod_{u \in S\left(q, b^{\prime}, d\right)}\left(1-t^{d} \prod_{i=1}^{n} \varpi\left(a_{i}\right)^{u_{i}\left(q^{d}-1\right)} \cdot g_{q^{d}}\left(u_{i}\left(q^{d}-1\right)\right)\right)^{\frac{1}{d}} \\
& =\prod_{d \in I} \prod_{u \in S\left(q, b^{\prime}, d\right)}\left(1-t^{d} \prod_{i=1}^{n} \varpi\left(a_{i}\right)^{u_{i}\left(q^{d}-1\right)} \cdot g_{q^{d}}\left(u_{i}\left(q^{d}-1\right)\right)\right)^{\frac{1}{d}} .
\end{aligned}
$$

Now let $u \in O\left(S\left(q, b^{\prime}, d\right)\right)$. Then note that $\omega\left(a_{i}\right)^{q}=\omega\left(a_{i}\right)$ and by Lemma 4.2 we know that for each of the $d$ points in the orbit of $u \in S\left(q, b^{\prime}, d\right)$ under $q$-action, the corresponding factor in (4.6) is the same. So we can remove the power $\frac{1}{d}$ in (4.6) by restricting $u$ to run over the set $O\left(S\left(q, b^{\prime}, d\right)\right)$. Therefore we get

$$
\begin{aligned}
& L^{*}\left(\chi_{1}, \ldots, \chi_{n}, f ; t\right)^{(-1)^{n-1}} \\
& \quad=\prod_{d \in I} \prod_{u \in O\left(S\left(q, b^{\prime}, d\right)\right)}\left(1-t^{d} \prod_{i=1}^{n} \varpi\left(a_{i}\right)^{u_{i}\left(q^{d}-1\right)} \cdot g_{q^{d}}\left(u_{i}\left(q^{d}-1\right)\right)\right) .
\end{aligned}
$$


But Lemma 2.1 applied to the finite field $\mathbf{F}_{q^{d}}$ gives

$$
g_{q^{d}}\left(u_{i}\left(q^{d}-1\right)\right)=\pi^{\sum_{i=1}^{n} \sigma_{p}\left(u_{i}\left(q^{d}-1\right)\right)} \prod_{j=0}^{d e-1} \Gamma_{p}\left(\frac{\left\langle p^{j} u_{i}\left(q^{d}-1\right)\right\rangle_{q^{d}-1}}{q^{d}-1}\right) .
$$

So by (4.7) and (4.8) we then get the desired formula (4.1).

Now from (4.1) we deduce that

$$
\begin{aligned}
& \operatorname{ord}_{q} \omega_{u} \\
& =\frac{1}{d} \cdot \operatorname{ord}_{q}\left(\pi^{\sum_{i=1}^{n} \sigma_{p}\left(u_{i}\left(q^{d}-1\right)\right)} \cdot \prod_{i=1}^{n} \varpi\left(a_{i}\right)^{u_{i}\left(q^{d}-1\right)} \prod_{j=0}^{d e-1} \Gamma_{p}\left(\frac{\left\langle p^{j} u_{i}\left(q^{d}-1\right)\right\rangle_{q^{d}-1}}{q^{d}-1}\right)\right) \\
& =\frac{1}{d} \cdot \operatorname{ord}_{q}\left(\pi^{\sum_{i=1}^{n} \sigma_{p}\left(u_{i}\left(q^{d}-1\right)\right)}\right) \\
& =\frac{1}{d(p-1)} \sum_{i=1}^{n} \sigma_{p}\left(u_{i}\left(q^{d}-1\right)\right) \\
& =\frac{1}{d(p-1)} \sum_{i=1}^{n} \frac{p-1}{q^{d}-1} \sum_{j=0}^{d e-1}\left\langle p^{j} u_{i}\left(q^{d}-1\right)\right\rangle_{q^{d}-1} \\
& =\frac{1}{d} \sum_{i=1}^{n} \frac{1}{q^{d}-1} \sum_{j=0}^{d e-1}\left\langle p^{j} u_{i}\left(q^{d}-1\right)\right\rangle_{q^{d}-1} .
\end{aligned}
$$

Since

$$
\left\langle p^{j} u_{i}\left(q^{d}-1\right)\right\rangle_{q^{d}-1}-\left\{p^{j} u_{i}\right\}\left(q^{d}-1\right) \equiv\left[p^{j} u_{i}\right]\left(q^{d}-1\right) \equiv 0 \quad\left(\bmod q^{d}-1\right)
$$

and note that $0 \leq\left\{p^{j} u_{i}\right\}\left(q^{d}-1\right) \leq q^{d}-1$, we have

$$
\left\langle p^{j} u_{i}\left(q^{d}-1\right)\right\rangle_{q^{d}-1}=\left\{p^{j} u_{i}\right\}\left(q^{d}-1\right) .
$$

So we get by (4.9) as well (4.10),

$$
\operatorname{ord}_{q} \omega_{u}=\frac{1}{d} \sum_{i=1}^{n} \frac{1}{q^{d}-1} \sum_{j=0}^{d e-1}\left\{p^{j} u_{i}\right\}\left(q^{d}-1\right)=\frac{1}{d} \sum_{j=0}^{d e-1} \sum_{i=1}^{n}\left\{p^{j} u_{i}\right\},
$$

as required. The proof of Theorem 4.3 is complete.

For any rational number $0 \leq r \leq n$, we define a number $h_{r}$ by

$$
h_{r}:=\sharp\left\{u \in S\left(\Delta, b^{\prime}\right) \mid \sum_{i=1}^{n} \sum_{j=0}^{d e-1}\left\{p^{j} u_{i}\right\}=d r\right\} .
$$

Therefore by Theorem 4.3 we derive the following result.

Theorem 4.4. Let $f(x)=\sum_{i=1}^{n} a_{i} x^{V_{i}} \in \mathbf{F}_{q}\left[x_{1}, x_{1}^{-1}, \ldots, x_{n}, x_{n}^{-1}\right]$ be a diagonal Laurent polynomial, and let $M=\left(V_{1}, \ldots, V_{n}\right)$ be the $n \times n$ matrix having $V_{i}$ as its $i$-th column for $1 \leq i \leq n$ satisfying $(p, \operatorname{det} M)=1$. Then for each rational number $0 \leq r \leq n$, the $p$-adic Newton polygon of the L-function $L^{*}\left(\chi_{1}, \ldots, \chi_{n}, f ; t\right)$ has a side of slope $r$ with horizontal length $h_{r}$.

Corollary 4.5. Let $f(x)=\sum_{i=1}^{n} a_{i} x^{V_{i}} \in \mathbf{F}_{q}\left[x_{1}, x_{1}^{-1}, \ldots, x_{n}, x_{n}^{-1}\right]$ be a diagonal Laurent polynomial, and let $M=\left(V_{1}, \ldots, V_{n}\right)$ be the $n \times n$ matrix having $V_{i}$ as its $i$-th column for $1 \leq i \leq n$. Let $(p, \operatorname{det} M)=1$. Then each of the following is true: 
(i) We have

$$
\begin{aligned}
& L^{*}(f ; t) \\
& =\prod_{d \in I_{0}} \prod_{u \in O(S(q, d))}\left(1-t^{d} \cdot \pi^{\sum_{i=1}^{n} \sigma_{p}\left(u_{i}\left(q^{d}-1\right)\right)}\right. \\
& \left.\quad \cdot \prod_{i=1}^{n} \varpi\left(a_{i}\right)^{u_{i}\left(q^{d}-1\right)} \prod_{j=0}^{d e-1} \Gamma_{p}\left(\frac{\left\langle p^{j} u_{i}\left(q^{d}-1\right)\right\rangle_{q^{d}-1}}{q^{d}-1}\right)\right),
\end{aligned}
$$

where $I_{0}$ is defined by $I_{0}:=\left\{d \in \mathbf{Z}^{+} \mid \exists u \in S(\Delta, 0)\right.$, such that $\left.d(q, 0, u)=d\right\}$ and $S(q, d):=S(q, 0, d)$.

(ii) If $u \in O(S(q, d))$ and $\omega_{u}$ is any one of the $d$ reciprocal roots of the corresponding factor in (4.11), then we have

$$
\operatorname{ord}_{q} \omega_{u}=\frac{1}{d} \sum_{j=0}^{d e-1} \sum_{i=1}^{n}\left\{p^{j} u_{i}\right\}
$$

(iii) For each rational number $0 \leq r \leq n$, the $p$-adic Newton polygon of the $L$-function $L^{*}(f ; t)$ has a side of slope $r$ with horizontal length $h_{r}$.

Finally we point out that if $f$ is degenerate, which means that $p \mid \operatorname{det} M$, then we can change it to the nondegenerate case by using some suitable transformations. For instance, let $f\left(x_{1}, x_{2}\right)=x_{1} x_{2}^{-1}+x_{1}^{-1} x_{2}^{1-p}$. Then $|\operatorname{det}(M)|=p$ and so $f$ is degenerate. First let $y_{1}=x_{1} x_{2}^{-1}, y_{2}=x_{2}$. Then it is an invertible transformation. Second let $y_{1}^{\prime}=y_{1}, y_{2}^{\prime}=y_{2}^{p}$. Finally let $z_{1}=y_{1}^{\prime}, z_{2}=y_{2}^{\prime} y_{1}^{\prime-1}$. This is also invertible. Hence we get $f\left(x_{1}, x_{2}\right)=z_{1}+z_{2}:=h\left(z_{1}, z_{2}\right)$ and

$$
L^{*}\left(\chi_{1}, \chi_{2}, f ; t\right)=L^{*}\left(\chi_{1}^{\prime}, \chi_{2}^{\prime}, h ; t\right)
$$

for some multiplicative characters $\chi_{1}^{\prime}, \chi_{2}^{\prime}$ of $\mathbf{F}_{q}$. But $h=z_{1}+z_{2}$ is nondegenerate. Therefore we can use Theorems 4.3 and 4.4 to compute the $L$-function $L^{*}\left(\chi_{1}^{\prime}, \chi_{2}^{\prime}, h ; t\right)$ (hence $L^{*}\left(\chi_{1}, \chi_{2}, f ; t\right)$ by $\left.(4.12)\right)$ and determine its $p$-adic Newton polygon.

\section{REFERENCES}

1. A. Adolphson and S. Sperber, Exponential sums and Newton polyhedra: Cohomology and estimates, Ann. of Math.(2) 130 (1989), 367-406. MR1014928 (91e:11094)

2. A. Adolphson and S. Sperber, $p$-Adic estimates for exponential sums, Lectures Notes in Math. 1454 (1990), 367-406. MR1094845 (92d:11086)

3. B. Dwork, On the zeta function of a hypersurface, Publ. Math. I.H.E.S. 12 (1962), 5-68. MR0159823 (28:3039)

4. B. Dwork, Normalized period matrices II, Ann. of Math.(2) 98 (1973), 1-57. MR0396580 $(53: 442 \mathrm{~b})$

5. B. Dwork, Bessel functions as p-adic functions of the argument, Duke Math. J. 41 (1974), 711-738. MR0387281(52:8124)

6. B.H. Gross and N. Koblitz, Gauss sums and the p-adic $\Gamma$-function, Ann. of Math.(2) 109 (1979), 569-581. MR:534763 (80g:12015)

7. S. Hong, Newton polygons of L-functions associated with exponential sums of polynomials of degree four over finite fields, Finite Fields Appl. 7 (2001), 205-237. MR.1803945 (2001j:11120)

8. S. Hong, Newton polygons for L-functions of exponential sums of polynomials of degree six over finite fields, J. Number Theory 97 (2002), 368-396. MR.1942966 (2003i:11116)

9. S. Hong, Newton polygons for L-functions of exponential sums of polynomials of degree five over finite fields, preprint. 
10. K. Ireland and M. Rosen, A classical introduction to modern number theory, GTM 84, Springer-Verlag, New York, 2nd edition, 1990. MR.1070716 (92e:11001)

11. B. Mazur, Frobenius and the Hodge filtration, Bull. A.M.S. 78 (1972), 635-667. MR0330169 $(48: 8507)$

12. A.M. Robert, A course in p-adic analysis, GTM 198, Springer-Verlag, New York, 2000. MR 1760253 (2001g:11182)

13. S. Sperber, Congruence properties of the hyper-Kloosterman sum, Compositio Math. 40 (1980), 3-33. MR 558257 (81j:10059)

14. S. Sperber, Newton polygons for general hyper-Kloosterman sums, Astérisque 119-120 (1984), 267-330. MR773095 (86i:11044)

15. S. Sperber, On the p-adic theory of exponential sums, Amer. J. Math. 108 (1986), 255-296. MR 833359 (87j:11055)

16. D. Wan, Newton polygons and congruence decompositions of L-functions over finite fields, Contemp. Math. 133 (1992), 221-241. MR1183982 (93i:11099)

17. D. Wan, Newton polygons of zeta functions and L-functions, Ann. of Math.(2) 137(1993), 249-293. MR1207208 (94f:11074)

18. D. Wan, Dwork's conjecture on unit root zeta functions, Ann. of Math.(2) 150 (1999), 867 -927. MR1740990 (2001a:11108)

19. D. Wan, Higher rank case of Dwork's conjecture, J. Amer. Math. Soc. 13 (2000), 807-852. MR.1775738 (2001f:11101a)

20. D. Wan, Rank one case of Dwork's conjecture, J. Amer. Math. Soc. 13 (2000), 853-908. MR:1775761 (2001f:11101b)

21. D. Wan, Variation of p-adic Newton polygons for L-functions of exponential sums, Asian J. Math. 8 (2004), 427-472. MR2129244 (2006b:11095)

22. R. Yang, Newton polygons of L-functions of polynomials of the form $x^{d}+\lambda x$, Finite Fields Appl. 9 (2003), 59-88. MR.1954784 (2003i:11180)

Mathematical College, Sichuan University, Chengdu 610064, People's Republic of CHINA

E-mail address: $\mathbf{s}-\mathbf{f}$. hong@tom.com

E-mail address: hongsf02@yahoo.com 